\title{
Parameterizing the Geometry and Visualizing the Lighting Method of Byzantine Church Domes
}

\author{
Wassim $\mathrm{Jabi}^{1}$ and Iakovos Potamianos ${ }^{2}$ \\ ${ }^{1}$ Welsh School of Architecture, Cardiff University, Cardiff, United Kingdom \\ jabiwdcardiff.ac.uk \\ ${ }^{2}$ School of Drama, Faculty of Fine Arts, Aristotle University of Thessaloniki, Thessaloniki, \\ Greece \\ ipota@thea.auth.gr
}

\begin{abstract}
This paper introduces a computer-based tool for the analysis of the geometry and the daylighting of Byzantine church domes to facilitate experimentation with a number of cases before any fieldwork is undertaken. Starting with a geometric derivation of the relationship between dome parameters, the digital tool builds an interactive three-dimensional model of a Byzantine church dome. The model allows the user to input the properties of the dome, the drum, any windows, and the slope of their sills. The model allows the user to define the dome using three different curvatures since such a case was identified in a Mistras church. A custom ray-tracing algorithm visualizes the path of light rays falling on the windowsills and their reflections within the dome. It was found that several parameters are interrelated and that an optimal set of proportions must be established to achieve the expected behavior of light within the dome.
\end{abstract}

Keywords: Byzantine domes, lighting simulation, parametric models, natural daylighting

\section{Introduction}

In many Byzantine churches the dome appears to be uniformly illuminated even though logically it could not be since the windows are located at the lower part of the dome and the sunrays always point downwards (Fig. 1). According to our studies this phenomenon was first posed and solved as a problem of solid geometry by Anthemius of Tralles presumably to achieve the unique lighting of the Hagia Sophia dome, his life masterpiece. Anthemius was an important geometer who was well versed in "catoptrics", a science, which captured the interest of mathematicians since classical antiquity. While the problem was clearly posed by Anthemius, there is no record that the combination of forms he invented was known or followed in subsequent churches. However, although later churches adopted a quite different geometry, the phenomenon of a highly lit dome persisted. In order to understand the manner in which a byzantine dome was lit, we have attempted several studies and simulations [1], [2], [3], [4], [5], of which the one appearing in this paper constitutes a new development. 


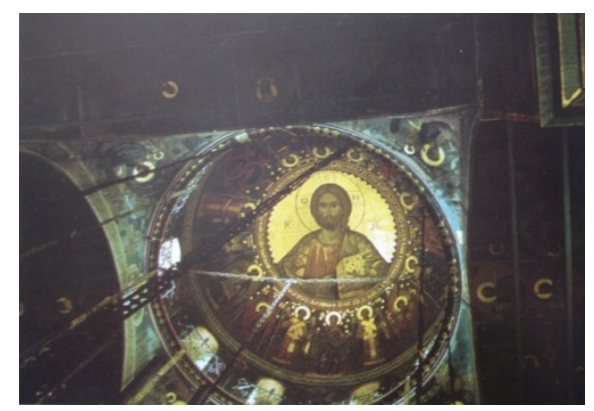

Fig. 1. Dome of the Iveron monastery church, Mount Athos, $11^{\text {th }} \mathrm{c}$.

The dome of Hagia Sophia of Istanbul, as has been established in our previous studies, had been designed as a combination of two curved mirrors (Fig. 2) of different curvature [1], [2]. These mirror designs were found in the architect's own extant writings [6]. The manner in which these two mirrors were combined has been shown in previous studies (Fig. 3), and the impression generated has been described by Procopius, the historian eye-witness of the effect. This hypothesis can be considered largely proven in terms of geometric, topographic and textual evidence [1].

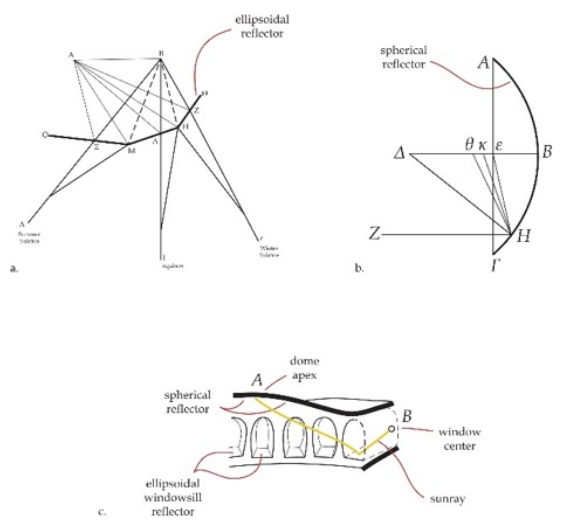

Fig. 2. a. Anthemius's ellipsoidal reflector, b. Anthemius's spherical reflector (i.e. the shallow dome itself), c. Sketch showing the location of the reflectors.

The most persistent questions posed, whenever these findings had been presented in academic fora, were the following: "Hagia Sophia is a unique case. How would domes of lesser church examples achieve a similar visual effect? Since their form is quite different than Hagia Sophia's and thereby could not have employed the same method of illumination, how did they achieve the constantly and uniformly luminous effect which is common among them?" 


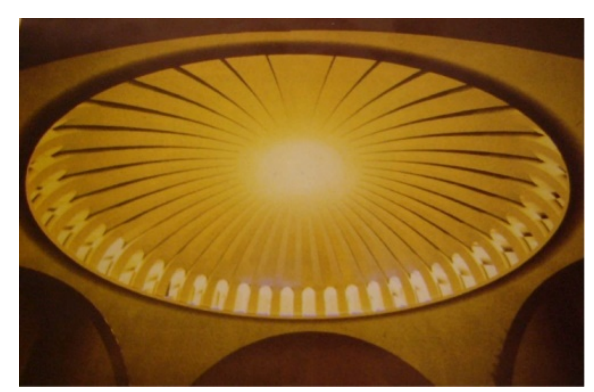

Fig. 3. Computer model of the original dome of Hagia Sophia with the two mirrors in place (from an earlier simulation).

\section{The case of other Byzantine churches}

Any attempt to answer the above questions tends to become particularly complex. The reason is that one is not dealing with a single building, which may be studied exhaustively from a number of different aspects. Instead, one has to investigate many different buildings of various eras. Therefore, the enormity of the problem requires a carefully planned strategy.

A highly and quite uniformly lit dome in lesser Byzantine church examples is a persistent phenomenon. This is not something expected or occurring in all domed churches. If there had been no lighting planning, a different portion of the dome would be lit depending on the position of the sun in the sky (Fig. 4) while the rest of the dome would remain darker. Instead, a Byzantine dome is uniformly lit and the brightest spot is usually found at the apex (Fig. 1). Therefore, it may be assumed that there must be a certain lighting system at work in the domes of lesser Byzantine churches as well. This system is what we set out to discover and to this purpose we thought that we could take advantage of the method of parametric modeling before we move into actual field work.

Following the construction of the parametric model one could then resort to surveys of existing domes. In typical surveys of Byzantine churches, published in various specialized articles, books, and dissertations, some basic, easily measurable variables are already available. Thus, one would have a significant amount of elementary measurements, (i.e. dome height, drum height, number and dimensions of dome windows) to begin with, before travelling to the monument site. One would then have to observe whether the dome of the monument is luminous, using existing photographs, and would manipulate the subtler variables of the parametric model in order to achieve the effect. Once the luminous effect had been achieved in the model one would already have a fair estimate of the variables that affect this visual impression. As a result, only those variables of the actual form would have to be measured that made sense in terms of the model. Strategically, this would be the most effective procedure. 


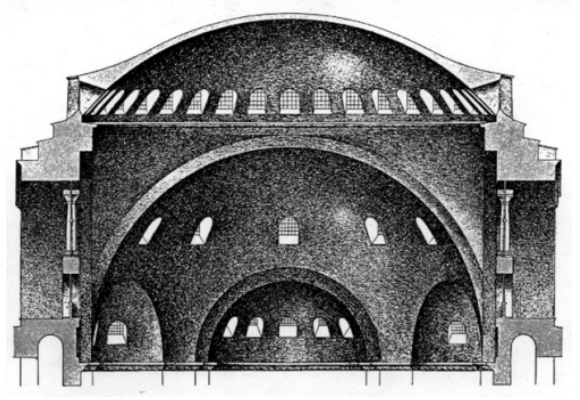

Fig. 4. Drawing of the original Hagia Sophia dome by E. Antoniades, showing a bright spot in the dome, located off center [8]. This image would be created if the lighting had not been carefully planned.

\section{Geometric principles of the parametric model}

The Byzantine church domes vary considerably. They are made up of various parts. Often, there is an upright standing tube-like part, which may be cylindrical or polygonal, called the "drum". On top of it a roughly semispherical dome is placed. On the drum several openings are pierced which differ in number from six to eight to twelve to forty. However, the closer one looks at that geometry the more variations one discovers to this basic scheme.

Below, certain general observations and principles are outlined which lie behind the construction of the model. First, the drum is not always of the same height and its proportions are related to the scale of the dome ensemble (i.e., the diameter of the dome in plan) as well as to the total height. A general proportioning rule is that a greater dome radius corresponds to a lower drum height. This rule has not yet been established as a strict mathematical proportional relationship.

In some cases, the drum disappears completely. This is the case, for instance, of the original dome of Hagia Sophia in Istanbul (Fig. 3), while in the church of Saints Sergius \& Bacchus - which served as a model for the design of the former - the drum is quite low. In the church of Hagia Eirini, of the same city, while a substantial drum is visible from the outside, in the interior the smooth curvature of the dome blends in with the drum and makes the drum invisible as if it were part of the dome. This difference is visible in most churches of later periods as well. While the drum is clearly articulated as a form from the exterior, it loses all articulation and blends in with the curvature of the dome as it is seen from the inside. Architecturally, the exterior and the interior seem as belonging to two different aesthetic approaches. This phenomenon requires a special investigation, which, however, is not the focus of this paper. What we might retain from this observation for now is that for some reason this sense of transition from one geometric form to another, so that the composite form appears unified, as seen from the inside, constituted an essential goal.

Second, the size of the windows piercing the drum depend on the drum's height. The taller the drum the greater the height of the windows and the slenderer their proportions become. This proportional relationship appears to be consistent. However, as 
we have already mentioned, the drum in some cases may not exist at all. In these cases, the window height acquires a proportional relationship to the sum of the circular segments length. Therefore, the possibility of inserting windows on the dome curvature should be incorporated in the model. The window height does have an effect on the manner in which light is reflected towards the dome. In a taller drum the rays seem to be able to be reflected more vertically while in a shorter drum the window header limits the reflected rays to more oblique angles.

Third, it has been noticed that the dome may not be necessarily semispherical. What has been observed in Hagia Sophia is that a very shallow curvature was employed. Such shallow curvatures have been observed in various other churches as well. Similar shallow curvatures were not limited to the dome but were employed also in vertical wall surfaces such as the one above the main gate of the church of Hagia Sophia, which seems to be responsible for the concentration of light so that the image of Christ, located on that surface, becomes suddenly lit at a certain distance [7]. Shallow curvatures, in general, seem to be connected to the impression of a uniformly lit dome. A shallow curvature has the ability to trap light and distribute it uniformly onto its surface, as long as light enters the curvature obliquely. This particular characteristic would render the shallow curvature invaluable to a dome designer. Therefore, our parametric model has the capability of incorporating a shallow dome curvature.

Fourth, a sunray passing through a dome window must somehow be reflected in order to reach the interior of the dome. The windowsill may provide such a surface. Anthemius especially designed such a surface for this location [1], [4]. However, this design, placed on the dome windowsill was conceived as a concave surface, which sloped inward. For the case of Hagia Sophia, of which the original dome had no drum, this windowsill design and slope was reasonable. For a dome with a drum, however, the same principle would tend to produce an outward sloping windowsill. We have found evidence of such treatment on the dome windowsills of the Monastery of Iveron church, which has a particularly bright and uniformly lit dome (Fig. 1). Based on these observations, the model has the capability of manipulating the windowsills by changing their slope and curvature.

Fifth, if not planned carefully, the reflected ray, once it reached the dome, would most probably hit it once and then be reflected downward to the floor. The point on which it hit would vary depending on the position of the sun in the sky. This means that a luminous point in the dome would appear at different positions while the rest of the dome would remain dim (Fig. 4). It appears that the only way to create a uniformly and constantly luminous dome would be to trap the light in it. This can happen if two conditions are met. First, if a shallow reflective surface for the interior of the dome itself is employed. Second, if a windowsill with a slope is employed so as to send the rays into the dome in such a way that further reflections continue to remain entrapped within the dome. Then, we could expect that most ordinary churches may incorporate not one but two or even three curvatures within the dome. The upper curvature of the dome may have to be deeper when a drum exists and be proportional to its height. The idea of a tri-partite dome was conceived based on the observation of an existing dome, of the Metropolis church of Mistras (Fig. 5). 


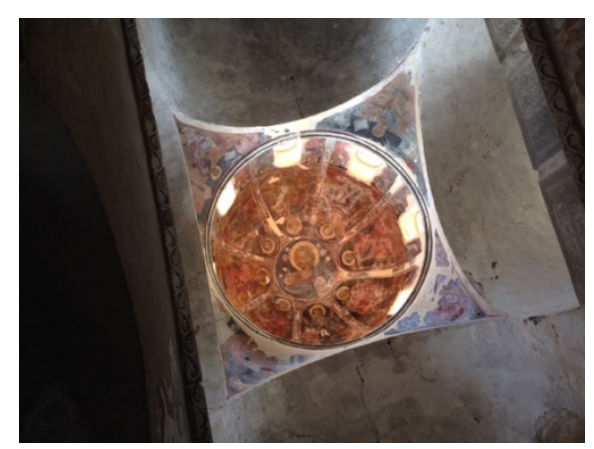

Fig. 5. Dome of Metropolis church, Mistras, $13^{\text {th }} \mathrm{c}$.

\subsection{Derivation of the geometric relationships}

The purpose of this section is to provide a geometric proof of the derivation of the two other radii (called middle dome radius and upper dome radius) given the first radius of the lower/main dome and a set of input parameters. This derivation forms the basis of the algorithms and the parametric calculations based on user input in the interactive graphical interface. Please refer to the diagram below for reference to the named vertices (Fig. 6).

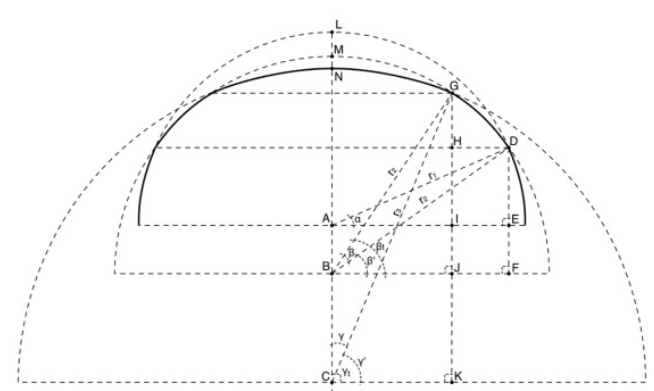

Fig. 6. Drawing showing the section of the dome which is composite of three circular curvatures. This drawing shows the fundamental variables of the dome introduced into the model.

Before we start the derivation, it is important to note that the naming of the domes (lower, middle, and upper), refers to the end result after its construction. These domes are shown in dashed lines in the figure above. The actual domes need to be vertically stacked in the opposite direction to achieve that end result (shown in a solid line). The lower dome arc has the smallest radius $(r l)$ and the highest vertical position for its center $(A)$, followed by the middle dome with a larger radius $(r 2)$ and a lower vertical position of its center $(B)$, and ending with the upper dome with the largest radius $(r 3)$ and the lowest vertical position of its center $(C)$.

We will mainly use Pythagoras's theorem which defines the relation in Euclidean geometry among the three sides of a right triangle. It states that the square of the hypotenuse (the side opposite the right angle) is equal to the sum of the squares of the 
other two sides. In addition, we will use trigonometric functions to derive the angles subtended by the arcs we are calculating. The calculated lengths and angles will allow us to geometrically create the necessary arcs of any desired dimension while maintaining consistent parametric relationships in the overall geometry.

\section{Given parameters}

The derivation assumes a set of given parameters:

1. $r_{l}$ is the radius of the lower dome. This is the main interior radius of the dome as perceived from the bottom at the "springing" point of the dome.

2. $A B$ is the vertical distance from the centre of the lower dome to the centre of the middle dome

3. $B C$ is the vertical distance from the centre of the middle dome to the centre of the upper dome.

4. $E D$ is the vertical height of the lower dome portion. Above that height, the dome arcs according to the middle dome radius $\left(r_{2}\right)$.

5. $H G$ is the vertical height of the middle dome portion. Above that height, the dome arcs according to the upper dome radius $\left(r_{3}\right)$.

\section{Derivation of the radius of the middle dome $(r 2)$}

$$
r_{I}^{2}=A E^{2}+E D^{2}
$$

Since $E D$ and $r l$ are given, we can derive $A E$.

$$
\begin{gathered}
A E=\sqrt{ }\left(r_{l}^{2}-E D^{2}\right) \\
B F=A E
\end{gathered}
$$

$A E$ is calculated in (2)

$$
F D=F E+E D
$$

Both $F E(A B)$ and $E D$ are given, so we can calculate $F D$.

$$
r_{2}{ }^{2}=B F^{2}+F D^{2}
$$

Since $B F$ and $F D$ have been calculated above in (3) and (4), we can derive $r_{2}$.

$$
r_{2}=\sqrt{ }\left(B F^{2}+F D^{2}\right)
$$

Derivation of the radius of the upper dome $(r 3)$

$$
\begin{gathered}
J G=J I+I H+H G \\
J I=A B, I H=E D \therefore J G=A B+E D+H G
\end{gathered}
$$

$A B, E D$, and $H G$ are given therefore we can derive $J G$. 


$$
r 2^{2}=J G^{2}+B J^{2} \therefore B J=\sqrt{ }\left(r 2^{2}-J G^{2}\right)
$$

Since $r 2$ and $J G$ have been calculated above, we can derive $B J$.

$$
C K=B J
$$

$B J$ is calculated in (9).

$$
\begin{gathered}
K G=K J+J I+I H+H G \\
K J=B C, J I=A B, I H=E D \therefore K G=B C+A B+E D+H G
\end{gathered}
$$

$B C, A B, E D$, and $H G$ are given, therefore we can derive $K G$.

$$
r 3^{2}=C K^{2}+K G^{2} \therefore r 3=\sqrt{ }\left(C K^{2}+K G^{2}\right)
$$

$C K$ and $K G$ have been calculated in (10) and (11) respectively, therefore we can derive $r 3$.

Thus, given the radius of the lower dome, the vertical position of the centres of the three domes and the vertical height of the lower and the middle dome portions, we can derive the radii of the lower and higher domes and construct a complex dome curvature made out of three arcs that can have a significantly lower apex $(N)$ compared to that of a single-radius dome $(L)$, or a double-radius dome $(M)$.

\section{Derivation of the angles}

The construction of the curves of the parametric dome also depend on discovering the subtended angles for the lower, middle, and upper dome. In the figure above, these angles are $\angle E A D(\alpha),<D B G(\beta)$, and $\angle G C N(\gamma)$ respectively. Using trigonometry, we can find these angles. Specifically, we can use the definition of the sin of an angle as the division of the opposite side divided by the hypotenuse in a right-angled triangle. Space limitations prevent us from including the full derivation in this paper.

\section{Computer implementation}

In order to investigate these hypotheses, we created a computer script within Autodesk 3ds Max 3D that generates a three-dimensional solid model of a dome (Fig. 7).

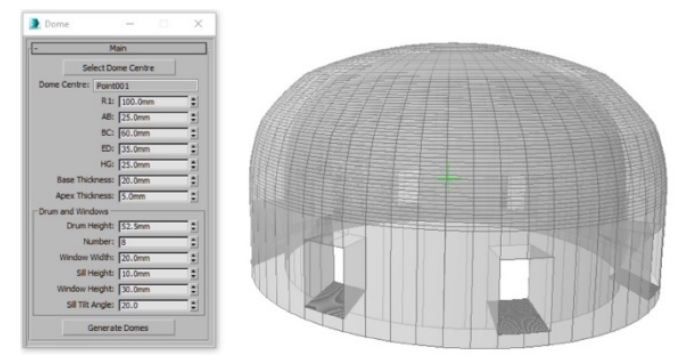

Fig. 7. Parametric dome model and associated input parameters. 
The parametric model presented here has been constructed with the capability of representing a dome created with three different arcs above the drum. The dome can acquire any radius and wall thickness. The lower and upper curvatures of the dome can vary in terms of their vertical height; thus creating different composite curvatures while maintaining a consistent geometric relationship between them. Any number of windows, of any width and height, can be incorporated into the drum or into the lower curvature of the dome. The windowsill can be made to slope inward or outward.

Once the dome has been created, we created a custom ray-tracing algorithm to simulate the behavior of light and reflection to visualize a representation of the sunrays as they hit the windowsill and reflect multiple times inside the dome (Fig. 8). This methodology is similar to the ones used for acoustic analysis [9]. The accuracy of the computer modelling has previously been verified [10]. In the image below, two subcases are presented simultaneously, one with a semispherical upper dome and another with a shallower upper dome in order to compare the manner in which light is reflected in each. The light is reflected in such a way that it washes the entire dome surface. This light behavior guarantees the uniform illumination of the entire dome.

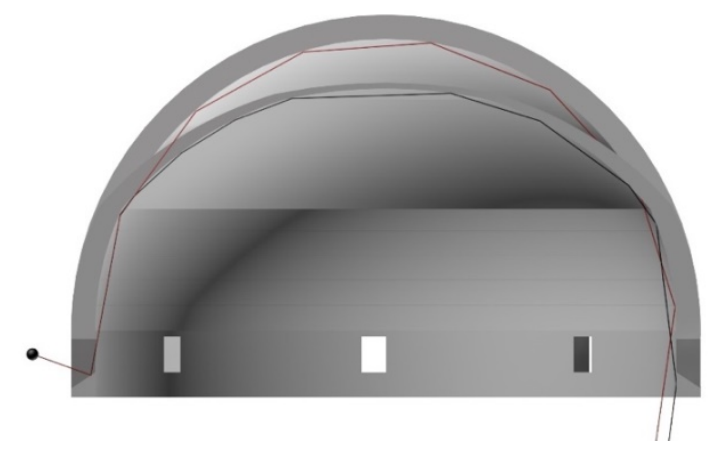

Fig. 8. Computer model comparing a semispherical and a tri-spherical dome

We decided that the most effective method to study the behavior of the multiple reflections within the dome was to calculate and visualize the line vectors that represent the direction of a sunray. It is important to note here that the built-in ray-tracing capabilities of the software environment were of no use to us at this stage as they only represent the final brightness and color of surfaces, but not the light ray vectors themselves which we needed. Furthermore, we also abandoned the use of the built-in physics simulation engine as reported in a previous paper. The reason for developing a ray-tracing algorithm rather than rely on the built-in physics engine is to eliminate data noise present in the physics simulation that takes into consideration the friction of surfaces, bounciness and other forces. In light-simulation, these forces do not exist. Thus, the enhanced script uses pure $3 \mathrm{D}$ vector geometry and rules of reflection to calculate the path of light as it reflects multiple times within the dome. While outside the scope of this paper, once the geometry is derived based on the mathematics of light, additional visual studies of texture and spatial perceptions can be undertaken following the examples in the project "Virtual Hagia Sophia" at MIRALab [11]. Simi- 
larly, high-dynamic-range (HDR) imaging can be used as a good method to present a similar range of luminance to that experienced through the human eye and has been used to better visualize light beams in existing buildings [12].

By varying the input parameters and re-running the ray-tracing algorithm, we were able to simulate multiple incidence angles and derive the appropriate parameters that can achieve the desired light path and therefore luminous effect through the entrapment of light within the dome (Fig. 9).

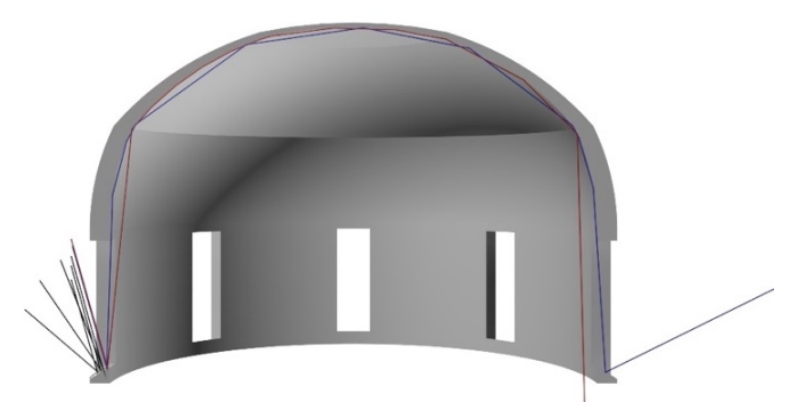

Fig. 9. Computer model of a dome with drum and a convex parabolic windowsill.

In addition to the parametric script that creates the main geometry of the dome, we enhanced our work with an additional script that allows us to generate and test the effect of parabolic surfaces. The script accurately creates a parabolic surface based on the location of a "focus" point and a "directrix" line. This three-dimensional parabolic surface can then be placed on top of the sill window and incorporated in the dome geometry. The axis of the parabola (i.e. its general direction) can be oriented in any angle. The orientation of the main axis of symmetry of the parabola determines the direction of reflections for any ray that falls on the exterior surface of the parabola and passes through its focus if extended to the other side of the parabola. However, parallel rays that hit the surface in other areas will reflect in different and non-parallel directions because they do not pass through the focus if extended. Using these scripts, we were able to simulate and visualize the behavior of several parallel light rays coming in at a steep angle as can be seen in (Fig. 9). The two rays in the above figure, the first exiting downwards on the right side (indicated in red) and the second exiting the window on the right side (indicated in blue), are close to each other and thus behave in a similar fashion. These two rays enter the dome space at a steeply vertical angle which causes them to reflect along the surface of the dome in a tangential matter. Other parallel rays that do not pass through the focus of the parabola are reflected in other directions - mainly back to the exterior of the dome. With the introduction of these additional scripts and the refined ray-tracing algorithm, we now have more accurate tools to further study different reflecting geometries and propose possible methods used to light the apex of the dome. 


\section{$5 \quad$ Summary of findings}

So far we have established the following:

1. In order to generate a light wash of the dome surface the rays must enter the concave surface as laterally to the dome curvature as possible. In a dome with a drum, this lateral introduction of the reflected ray corresponds to the vertical direction.

2. The taller the drum the more possible it is for the reflected ray to enter the dome at a more vertical angle.

3. The variable that is most important for the upward lateral direction of the reflected sunray is the slope of the windowsill of the dome windows. In a dome with a drum the windowsill must slope outwards while in the absence of a drum it must slope inwards.

4. In order for the reflected sunrays to acquire the same upward direction regardless of the position of the sun in the sky, the windowsill must be curved and most probably convex, acquiring the form of a parabola.

5. Both a semispherical dome as well as a tri-spherical one will generate consecutive reflections within it, given the appropriate windowsill shape.

6. In a tri-spherical dome the transition from one curvature to the next must be as gradual as possible in order for the reflections to follow the dome curvature closely.

7. The difference in a tri-spherical dome appears to be that more reflections are concentrated toward the upper parts of the dome.

8. Various parameters are interrelated. The height of the drum to the number and radius of the curvatures of the dome as well as to the rotation of the central axis of the convex parabolic windowsill.

9. Every sunray that falls in the direction of the focus of the parabola is reflected upwards within the dome and begins travelling along its curvature.

10. The closer the first reflection is to the vertical the greater the entrapment of the reflections within the dome.

11. Most of the sunrays that do not align with the parabola focus are reflected back out. Therefore, this type of curvature creates a highly selective and exclusive windowsill.

\section{Conclusions and future work}

Byzantine church domes are paradoxically brighter than all surrounding surfaces. This phenomenon has been investigated in previous work for the case of the dome of Hagia Sophia of Istanbul. The lighting of this dome had been resolved by the combination of two reflectors designed by Anthemius. In order to understand the dome design methods employed in lesser church examples, where a luminous dome effect is achieved, a parametric model was constructed which gave us the opportunity to experiment with a number of parameters influencing the behavior of light within the dome before we test any particular church case. It has been found that several of the 
parameters are interrelated, as for instance, the height of the drum, the slope of the windowsill and the height of the three curvatures of the dome. Depending on the various forms constituting the dome an optimal set of proportions must be established for the behavior of light within the dome. It is probable that there is a precise mathematical relationship governing these factors. This is an area to be investigated in future work. Such an investigation is valuable along with archaeological evidence to the restoration of domes of Byzantine churches of various periods. Contingent on the availability of funding, we plan to use advanced laser scanning and photogrammetry techniques on site to document the geometry of selected domes and their windowsills to verify our parametric model findings. Given the height of windowsills, we anticipate the need for aerial-based photogrammetry (e.g. using quadcopter drones equipped with cameras and 3D laser scanning equipment). Ultimately, we would like to study the experiential qualities achieved through the manipulation of light and its interaction with the architecture.

\section{$7 \quad$ References}

1. I. Potamianos, "Light into Architecture: The Evocative Use of Natural Light as Related to Liturgy in Byzantine Churches," Ph.D. dissertation, Doctoral Program in Architecture, University of Michigan, Ann Arbor, 1996.

2. W. Jabi and I. Potamianos, "A Parametric Exploration of the Lighting Method of the Hagia Sophia Dome" in Proc. 2006 7th International Conference on Virtual Reality, Archaeology and Intelligent Cultural Heritage, pp. 257-265.

3. W. Jabi and I. Potamianos, "Geometry, Light, and Cosmology in the Church of Hagia Sophia", International Journal of Architectural Computing, vol. 5(2), 2007, pp. 303-319.

4. W. Jabi and I. Potamianos, "A Parametric Exploration of the Lighting Method of the Hagia Sophia Dome," in Proc. 2006 The $7^{\text {th }}$ International Symposium on Virtual Reality, Archaeology and Cultural Heritage, pp. 257-265.

5. W. Jabi and I. Potamianos, "Interactive Parametric Design and the Role of Light in Byzantine Churches," in Proc. 2006 24th eCAADe Conf., pp. 798-803.

6. G. L. Huxley, Anthemius of Tralles: A Study of Later Greek Geometry, Cambridge, MA: Eaton Press, 1959.

7. G. Mathew, Byzantine Aesthetics, New York: Viking Press, 1964.

8. E. Antoniades, Ekphrasis tes Hagias Sophias, 1905.

9. J. Turner and T.W. Hall, "An Application of Geometric Modeling and Ray Tracing to the Visual and Acoustical Analysis of a Municipal Open-air Auditorium," in Proc. 1990 ACADIA '90, pp. 173-185.

10. T. Taengchum and S. Chirarattananon, "Ray Tracing Method of Light through Rectangular Light Pipe with Bends,” Energy Procedia, vol. 79(2), 2015, pp. 791-798.

11. A. Foni, G. Papagiannakis, N. Cadi-Yazli and N. Magnenat Thalmann, "Time-Dependant Illumination and Animation of Virtual Hagia-Sophia," International Journal of Architectural Computing (IJAC) (IF: 1.144), Multi-Science Publishing, vol. 5(2), 2007, pp. 284301.

12. J. Happa, A. Artusi, S. Czanner and A. Chalmers, "High Dynamic Range Video for Cultural Heritage Documentation and Experimental Archaeology," In Proc. 2010 VAST'10 the 11th International Symposium on Virtual Reality, Archaeology and Cultural Heritage. 